N López Vidales, L Gómez Rubio, D Vicente Torrico (2017): “La información territorial en la radio y la televisión de ámbito nacional en España y su contribución a la imagen de las comunidades autónomas". Revista Latina de Comunicación Social, 72, pp. 649 a 666.

\title{
La información territorial en la radio y la televisión de ámbito nacional en España y su contribución a la imagen de las comunidades autónomas
}

\section{Regional news in Spain's national radio and television and their contribution to the image of Spain's autonomous communities}

\begin{abstract}
Nereida López Vidales [CV] (D) http://orcid.org/0000-0002-6960-6129 C
http://scholar.google.es/citations?user=d-h-uasAAAAJ\&hl=es Profesora Titular de Periodismo.

Universidad de Valladolid (España) nereida.lopez@hmca.uva.es

Leire Gómez Rubio [CV] (1) http://orcid.org/0000-0002-6436-0297

http://scholar.google.es/citations?user=dufyKbUAAAAJ\&hl=es Profesora Ayudante-Doctora de

Periodismo. Universidad de Valladolid (España) leire.gomez@hmca.uva.es
\end{abstract}

David Vicente Torrico [CV] 1 http://orcid.org/0000-0003-0379-6086

https://scholar.google.es/citations?user=3DMiJ-0AAAAJ\&hl=es Personal Investigador en

Formación. Universidad de Valladolid (España) david.vicente.torrico@uva.es

\begin{abstract}
s
[ES] Introducción: Los informativos de radio y televisión de las cadenas generalistas nacionales son ventanas a las que cada día se asoma la audiencia para informarse, convirtiéndose ambos medios en herramientas transmisoras de información y de la imagen del territorio de procedencia de las noticias emitidas en sus servicios informativos. El objetivo principal de esta aportación es el análisis de la información territorial presente en los informativos nacionales de las cadenas de radio y televisión generalistas para aproximarnos a la imagen que proyectan sobre las distintas comunidades autónomas a la sociedad española. Metodología: Se ha optado por una combinación metodológica, cuantitativa y cualitativa, basada en el análisis de contenido, que facilitará la obtención de datos pertinentes sobre las unidades de análisis, las emisoras de radio y de televisión con mayores índices de audiencia en España en 2017: Cadena SER, Onda Cero, COPE, RNE, Telecinco, Antena 3 y TVE. La muestra se compone de 848 piezas procedentes de los informativos emitidos de 14 a 15 horas, de lunes a viernes, en los meses de enero y febrero de 2017. Resultados y conclusiones: La información territorial ofrecida por los informativos de radio y televisión nacionales está dominada por tres comunidades autónomas - Cataluña, Andalucía y Madrid-, siendo las noticias de política, sociedad y sucesos las que copan la información territorial emitida.
\end{abstract}


[EN] Introduction: National radio and television news programmes are daily sources of information for an increasing audience and, thus, are important tools in the shaping of the image of the Spanish autonomous communities that are represented in their news stories. The main objective of this article is to analyse the representations of the different Spanish autonomous communities in the regional news of mainstream national radio and television news programmes. Methods: The study combines quantitative and qualitative content analyses, which facilitate the collection of relevant data about the units of analysis: radio and television channels with the highest ratings in Spain in 2017: Cadena SER, Onda Cero, COPE, RNE, Telecinco, Antena 3 and TVE. The sample is composed of 848 news items selected from news programmes broadcast on weekdays, from 14:00 to 15:00 hours, in January and February 2017. Results and conclusions: The regional news stories offered by national radio and television news programmes are dominated by three autonomous communities -Catalonia, Andalusia and Madrid- and three news categories: politics, society and accidents and crime.

\section{Keywords}

[ES] Información territorial; Servicios informativos; Radio; Televisión; Imagen difundida.

[EN] Regional news; news services; radio; television; media representations.

\section{Contents}

[ES] 1. Introducción. 1.1. Objetivos e hipótesis. 2. Metodología. 3. La presencia territorial de la información diaria de radio y televisión. 3.1. Análisis de los resultados obtenidos en las cadenas de radio. 3.1.1. Hora 14, Cadena Ser. 3.1.2. Mediodía COPE, Cadena COPE. 3.1.3. Noticias Mediodía, Onda Cero Radio. 3.1.4. Diario de las 2, Radio Nacional de España. 3.2. Análisis de los resultados obtenidos en los canales de televisión. 3.2.1. Informativos Telecinco. 3.2.2. Antena 3 Noticias. 3.2.3. Telediario 1. 4. Discusión de resultados y conclusiones. 5. Notas. 6. Bibliografía.

[EN] 1. Introduction. 1.1. Objectives and hypothesis. 2. Methods. 3. The daily presence of regional news in radio and television newscasts. 3.1. Results about radio newscasts. 3.1.1. Hora 14, Cadena Ser. 3.1.2. Mediodía COPE, Cadena COPE. 3.1.3. Noticias Mediodía, Onda Cero Radio. 3.1.4. Diario de las 2, Radio Nacional de España. 3.2. Results about television newscasts. 3.2.1. Informativos Telecinco. 3.2.2. Antena 3 Noticias. 3.2.3. Telediario 1. 4. Discussion and conclusions. 5. Notes. 6. References.

Traducción de CA Martínez-Arcos (Doctor en Comunicación, Universidad de Londres)

\section{Introducción}

Los servicios informativos de las cadenas de radio y televisión de carácter generalista y ámbito nacional siguen siendo hoy en día las principales fuentes de información de los españoles, lo que induce a pensar que su influencia sobre la opinión pública en general es muy importante [1]. En el caso de la radio, la audiencia de las cadenas generalistas de ámbito nacional -RNE, COPE, SER y Onda Cero- se situó, de lunes a viernes, en 10.427.000 oyentes en enero de 2017, al tiempo que, sólo los informativos del mediodía, alcanzaron el medio millón de oyentes diarios entre las cuatro cadenas citadas. En lo que a la televisión se refiere, el mes de febrero concluyó con un "empate técnico" entre los informativos de La 1 -con 2.065.000 espectadores- y Telecinco -con 2.061.000en la ponderación de las ediciones de mediodía-sobremesa y noche, mientras que el informativo de Mediaset es líder en la edición de la noche, de lunes a domingo, y La 1 lidera el ranking en la edición de sobremesa de lunes a viernes, con una cuota de pantalla del $15,9 \%$, seguido por Telecinco $(13,8 \%)$, Antena $3(13,1 \%)$, La Sexta (12,7\%) y Cuatro (11\%) (Barlovento Comunicación, 2017). 
Los datos de audiencias referidos ponen de manifiesto que el público sigue eligiendo los informativos generalistas para informarse. Son, por tanto, ventanas de difusión de mensajes objetivos y subjetivos de primera magnitud para tratar de comprender muchos procesos cognitivos, perceptivos y comunicativos sobre la realidad social y sus protagonistas, que escuchan y visualizan millones de ciudadanos a diario.

Es una realidad que los programas de noticias suponen un elemento nuclear en la programación generalista. Como señalan Delgado y Prado el noticiario es un género ancla para las audiencias, uno de los principales elementos de la política de imagen de marca de una cadena y de fidelización de la audiencia. En la actualidad, además, y aunque algunos noticiarios conservan el espíritu original y hacen su selección de noticias con criterio preferencial para las hard news, las soft news están ganando terreno como tendencia general y cada vez es más frecuente el amarillismo, la espectacularización y los contenidos propios de las revistas de papel satinado (2010:11).

En el actual contexto globalizado, la sociedad mediática se define como aquella cuya principal fuente de significados es proporcionada por los medios de comunicación. Son el punto de acceso al núcleo de la sociedad (Couldry \& Hepp, 2012:97). Pero los medios de comunicación no transmiten toda la información disponible. Seleccionan solo una parte de esa información (Igartua \& Muñiz, 2007:49), producen relatos que, como todos los relatos, simplifican el espacio político y social que describen (Couldry \& Hepp, 2012:92) y los atributos que son destacados por los medios de comunicación quedan a su vez destacados en la mente del público (McCombs, 2004:164), con lo que aquellas cosas que los medios ignoran, muy bien pudieran no existir (Pitts, 2001:223) ya que son los medios los que establecen la agenda pública y dirigen el diálogo social. Lo que sabemos de nuestra sociedad, incluso acerca del mundo en que vivimos, lo sabemos por los medios de comunicación (Luhmann, 1996:2).

Para la mayoría de los ciudadanos, casi todo el conocimiento de lo político y social es construido a través de los medios (Crespo, Moreno \& Delgado, 2003:13-14) por lo que aquello que ocurre en las redacciones acerca de lo que se considera noticia en base a valores como la importancia, el interés, número de afectados o proximidad geográfica (Wolf, 1987: 222-248) es de vital trascendencia para construir la representación social y el imaginario colectivo. Como señala Wagner (1994:199) las representaciones sociales suelen definirse como un conjunto de creencias, imágenes, metáforas y símbolos compartidos por un grupo, comunidad, sociedad o cultura y es aquí donde cobra especial relevancia la realidad más conveniente que los ciudadanos pueden y saben percibir porque lo no referido por los medios se puede traducir como los temas más inconvenientes de la realidad y por lo mismo carecen de opinión colectiva (Lozano, Piñuel \& Gaitán, 2010:4). En última instancia, lo que creemos saber es algo de lo que hemos oído hablar, algo que nos ha sido contado, cuya verdad se sostiene por la confianza en instancias, autoridades, testigos y expertos (Lozano et al, 2010:13).

Dentro de la relación entre medios de comunicación y audiencia, y en concreto sobre la imagen de la realidad (o realidades) que los primeros proyectan sobre el público, tenemos en cuenta, además, la idea de Zavala (2003) en el sentido de que la imagen es como un espejo donde se proyecta, se reconoce y se recrea la propia identidad a través del ejercicio de sensibilidad (citado en Cañamero, 2014:353). Y es que las informaciones vertidas por los medios contribuyen "a difundir representaciones que producen significados (ideas, visiones, tópicos), sobre otros territorios, pueblos y culturas, construyendo una mirada sobre ellos" (Escudero, 2005:223). Y no solo proporcionan información e imagen nacional, sino también de proximidad a través de las diferentes noticias territoriales que cada uno de estos medios incluye en sus espacios informativos, y que adquieren especial relevancia, ya que "una sociedad local sin información de proximidad no puede reflejar su propia pluralidad o diversidad" (Chomón, 2011:21). Estas noticias territoriales, además, han tenido 
durante décadas un valor añadido en aquellas comunidades autónomas que han contado de forma tardía con televisiones de proximidad, tanto autonómicas como locales.

La exploración bibliográfica realizada con carácter previo al planteamiento de esta investigación revela que las publicaciones existentes acerca de nuestro objeto de estudio son escasas, e incluso inexistentes para la mayoría de las comunidades autónomas. Entre las que sí cuentan con estudios relevantes de este tipo se encuentran Andalucía, con el estudio realizado por el Instituto Oficial de RTVE para el Consejo Audiovisual de Andalucía en 2009, y otro anterior, titulado "La imagen de Andalucía en los informativos de televisión en España" (Ruiz Morales, 2007), financiado por la Junta de Andalucía; Navarra, que en 2006 publicó el tercer informe sobre "La imagen de Navarra en los medios de comunicación" (Sánchez-Aranda y García-Ortega, 2006); Extremadura, con "Extremadura representada en los medios de comunicación. Intertextualidad y estereotipos" (Cañamero, 2014); Galicia, "Galicia en cartel. A imaxe de Galicia na cartelaria turística" (VVAA, 2005), y Castilla y León, con un estudio sobre la imagen de Castilla y León en la prensa nacional de referencia (Berrocal, Campos \& Martínez, 2016).

Tomando como referencia los planteamientos de los estudios mencionados, hemos querido continuar con la línea de aproximación hacia cómo contribuyen los espacios informativos con sus noticias, y en menor medida sus comentarios, a la construcción del imaginario colectivo sobre estas unidades políticas, geográficas y sociales tan amplias que son las comunidades autónomas. En el presente trabajo exponemos, por tanto, los resultados de una investigación llevada a cabo sobre la información que la radio y la televisión nacionales ofrecen, a través de sus servicios informativos diarios, acerca de las distintas regiones del Estado, con el objetivo de ver si realmente estos medios contribuyen a la conformación de una determinada imagen autonómica. Y es que a pesar de que el mundo digital y las nuevas tecnologías han abierto una nueva senda donde el conocimiento se comparte y la creación de contenidos en Internet es un valor al alza (López-Vidales, Gómez-Rubio \& Tapia-López, 2015), los medios generalistas convencionales cuentan con una notable aceptación por parte de la población española.

\subsection{Objetivos e hipótesis}

Como ya se ha referido, la pretensión fundamental de este estudio es aproximarnos a la imagen que los medios de comunicación audiovisuales, radio y televisión concretamente, proyectan acerca de las diferentes comunidades autónomas y su población a través de las noticias difundidas en sus informativos diarios. Para ello, se analizan los servicios informativos de prime time (la $1^{\text {a }}$ edición), con especial atención a las noticias que se generan dentro y fuera de cada uno de los territorios del Estado, la naturaleza de aquellas informaciones que se generan y el papel que las autonomías juegan en la actualidad informativa nacional. La recogida de estos datos, nos permitirá establecer, de forma intrínseca, una radiografía comparativa entre las distintas comunidades autónomas en base a la información que generan y protagonizan, a los valores que se relacionan con esas noticias, al formato que se utiliza para exponerlas desde el punto de vista periodístico, y junto a ello, al enfoque y a los recursos que se emplean para presentar y difundir la noticias.

Para nuestro análisis partimos de la hipótesis general de que la información territorial ofrecida por los servicios informativos de las emisoras generalistas de radio y televisión de ámbito nacional es reducida y está dominada por aquellas autonomías con mayor peso político, económico y social en el conjunto del Estado, como son los casos de Madrid y Cataluña, mientras que el resto de regiones solo aparecen en estos informativos cuando ocurre algún suceso de alto impacto social.

\section{Metodología}


Para la realización de nuestro estudio [2] hemos optado por una combinación metodológica, cuantitativa y cualitativa, basada en el análisis de contenido que nos facilitará la obtención de datos pertinentes sobre las unidades de análisis a partir de la confección de una ficha en la que estarán presentes todas las variables de observación que destacamos como fundamentales para lograr nuestro objetivo. La muestra de análisis se compone de un total de 848 piezas audiovisuales, procedentes de los espacios informativos emitidos de lunes a viernes en horario de prime time, al mediodía, tanto en las cadenas de radio nacionales y generalistas como en las de televisión (35 espacios completos), siendo estas Cadena SER, Cadena COPE, RNE 1, Onda Cero, TVE1, Antena 3 TV y Tele 5. Los horarios de emisión escogidos son: de 14 a 15 horas para los servicios informativos radiofónicos y de 15 a 16 horas para los informativos televisivos [3]. El periodo de análisis comprende los meses de enero y febrero de 2017 según una selección aleatoria de unidades que tiene en cuenta los cinco días de la semana.

Con esta selección de días no consecutivos hemos querido evitar la persistencia de algunas noticias en el tiempo, tales como la crisis del PSOE o Unidos Podemos, las medidas adoptadas por Donald Trump como presidente de los Estados Unidos o los diversos juicios por corrupción política que se celebran en los últimos meses en España. Los programas analizados han sido escuchados y visualizados a través de las páginas webs de sus respectivas cadenas.

La justificación de la muestra proviene de la necesidad de fijar unos objetos de investigación de cobertura nacional y amplios índices de audiencia de manera que esté asegurada la recepción de la información que transmiten en todos los hogares del Estado, y proporciona una selección de material de análisis en línea con estudios de similares características (Humanes, 2001; López Téllez \& Cuenca García, 2005).

En nuestro análisis observaremos especialmente las noticias de cada informativo [4], aunque tendremos en cuenta también los comentarios presentes en algunos de los espacios seleccionados por si fueran de interés relevante para la interpretación final [5]. La ficha creada ad hoc para este estudio contempla los siguientes aspectos generales: codificación de la unidad, aspectos formales, temas y protagonistas; análisis específico de las noticias objeto de la investigación; y valoraciones generales sobre el orden, la exposición de la información, el enfoque, la profundidad y la percepción final del contenido. De este modo, en el análisis cuantitativo nos fijaremos en: la cadena de emisión; el espacio informativo; la duración; el tiempo total de la información; el número total de informaciones, entrevistas (y otros géneros si los hubiere); listado de titulares y duración; listado de noticias y otros formatos de información (por secciones) y duración de cada unidad; titulares y noticias dedicadas a otras entidades territoriales y su duración. En el análisis cualitativo nos centraremos en: las noticias territoriales (de cada comunidad autónoma); la cadena en la que se ha difundido; el formato; la duración; si está en titular; el orden que ocupa dentro del minutado; la sección y el área informativa en la que se incluye; los recursos utilizados; la percepción positiva, negativa o neutra de la noticia en función del lenguaje utilizado; el enfoque periodístico; la adjetivación, y por último, la profundidad de la información emitida.

Por último, para la interpretación y valoración final del análisis serán pertinentes también aspectos relacionados con el modo en el que se presentan las noticias, la relevancia que se les otorga dentro del espacio informativo, la valoración de las acciones que se presentan y el incierto sentido de la oportunidad periodística de presentar al público una determinada información excluyendo otras.

\section{La presencia territorial en la información nacional diaria de radio y televisión}

La exposición de los resultados de la investigación la presentamos teniendo en cuenta la audiencia general de cada uno de los medios de comunicación analizados. En primer lugar, se muestran los 
datos correspondientes a los informativos radiofónicos y después, los relativos a los informativos de televisión.

\subsection{Análisis de los resultados obtenidos en las cadenas de radio}

Las cadenas de radio generalistas españolas de cobertura nacional cuentan en sus parrillas con desconexiones territoriales diarias para informar sobre la actualidad de cada territorio a los ciudadanos de las respectivas comunidades autónomas. Estos espacios de noticias suelen desarrollarse inmediatamente antes del informativo nacional, sobre las 13 horas, y en él se recoge información de proximidad, local y específica, donde los protagonistas son las administraciones, grupos y ciudadanos de cada territorio. A las 14 horas comienzan generalmente en todas las emisoras los informativos de ámbito nacional por lo que es lógico pensar que en estos últimos espacios los hechos que prevalezcan sean los de influencia más general frente al interés más particular de los espacios de desconexiones territoriales. Estamos de acuerdo, entonces, en que la presencia de la información autonómica o territorial en estos servicios informativos será reducida, pero la realidad plurinacional del Estado no hace viable la elaboración de un espacio de noticias de una hora de duración sin hacer referencia a hechos y aconteceres de las distintas comunidades autónomas que marcan, en muchos casos, la agenda política, económica y cultural de todo el país.

\section{Gráfico 1. Peso informativo de las noticias territoriales en las cadenas de radio}

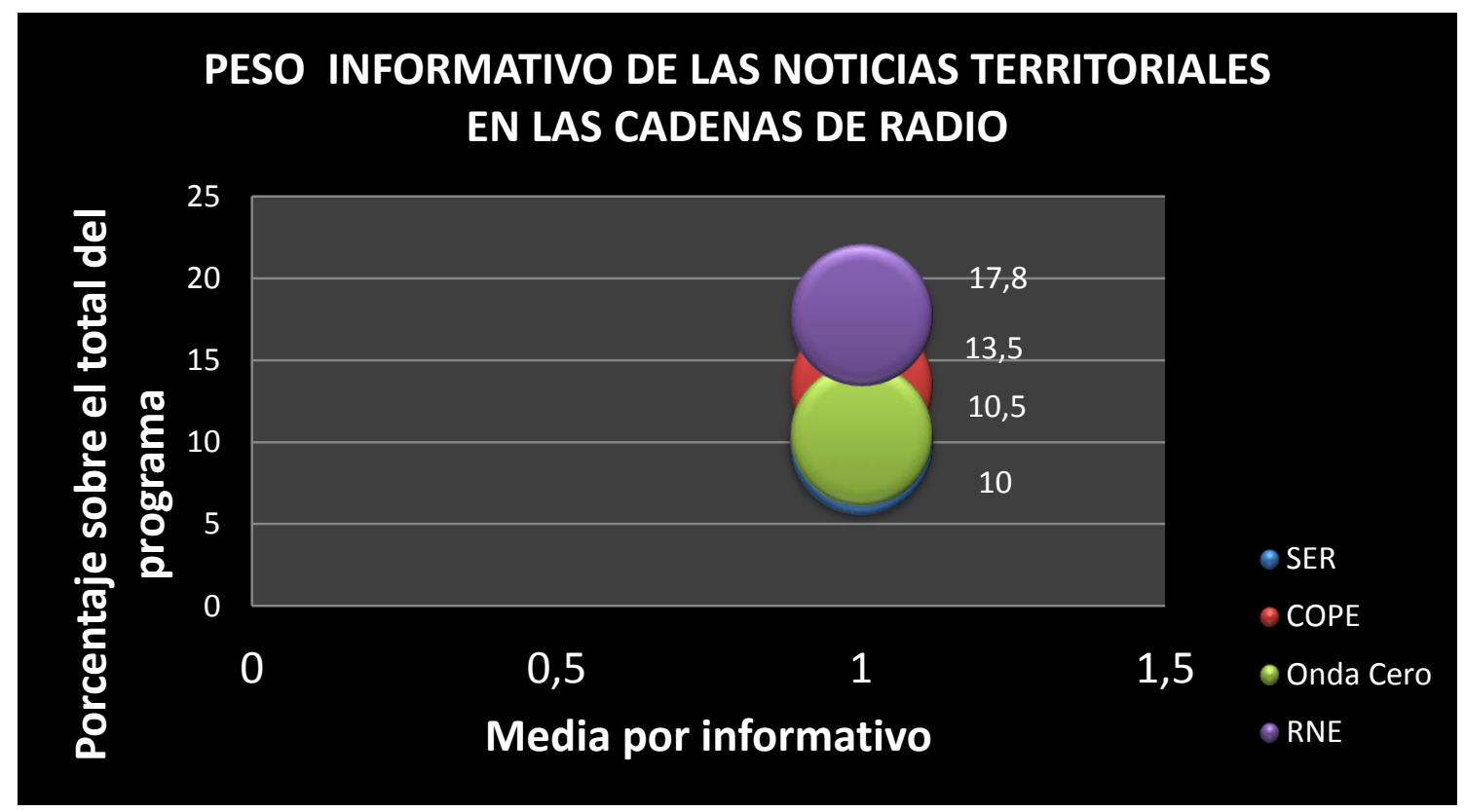

Fuente: elaboración propia.

De las emisoras de radio objeto de estudio -SER, COPE, Onda Cero y RNE-, la emisora pública es la que cuenta, dentro de sus informativos, con un mayor número de noticias referidas a las comunidades autónomas. En total, durante el periodo analizado se contabilizan en esta emisora 45 piezas sobre noticias territoriales, frente a las 22 ofrecidas por COPE, las 20 de Onda Cero y las 17 de SER, lo que supone una media de 9 informaciones de este tipo diarias, y de casi 21 entre todas las cadenas. Este dato es consecuente con el hecho que, de los cuatro, el espacio informativo de RNE es el que tiene una mayor duración, en torno a 60 minutos, mientras que el informativo de la Cadena SER apenas alcanza los 30, debido a la duración de la desconexión territorial y espacios publicitarios. 
Por comunidades autónomas, Cataluña es el territorio al que mayor espacio dedican, con un total de 35 piezas y más de 57 minutos de emisión durante la semana analizada. Aunque su presencia en los espacios informativos es constante, el 2 de febrero es la jornada que cuenta con un mayor número de informaciones [6], un total de 8, con 18:15 minutos en antena entre todas las emisoras. La mayoría de las informaciones se refieren al enfrentamiento político entre la Generalitat y el Gobierno central, fruto del proceso independentista impulsado por el equipo de Puigdemont, y a las consecuencias judiciales derivadas del referéndum celebrado el 9 de noviembre de 2014. Esto provoca que la sección de política sea la que ocupe un mayor espacio en los servicios informativos analizados, con una duración media de 12:30 y 6 noticias diarias.

\section{Gráfico 2. Tiempo dedicado a la información territorial en la radio generalista de ámbito nacional}

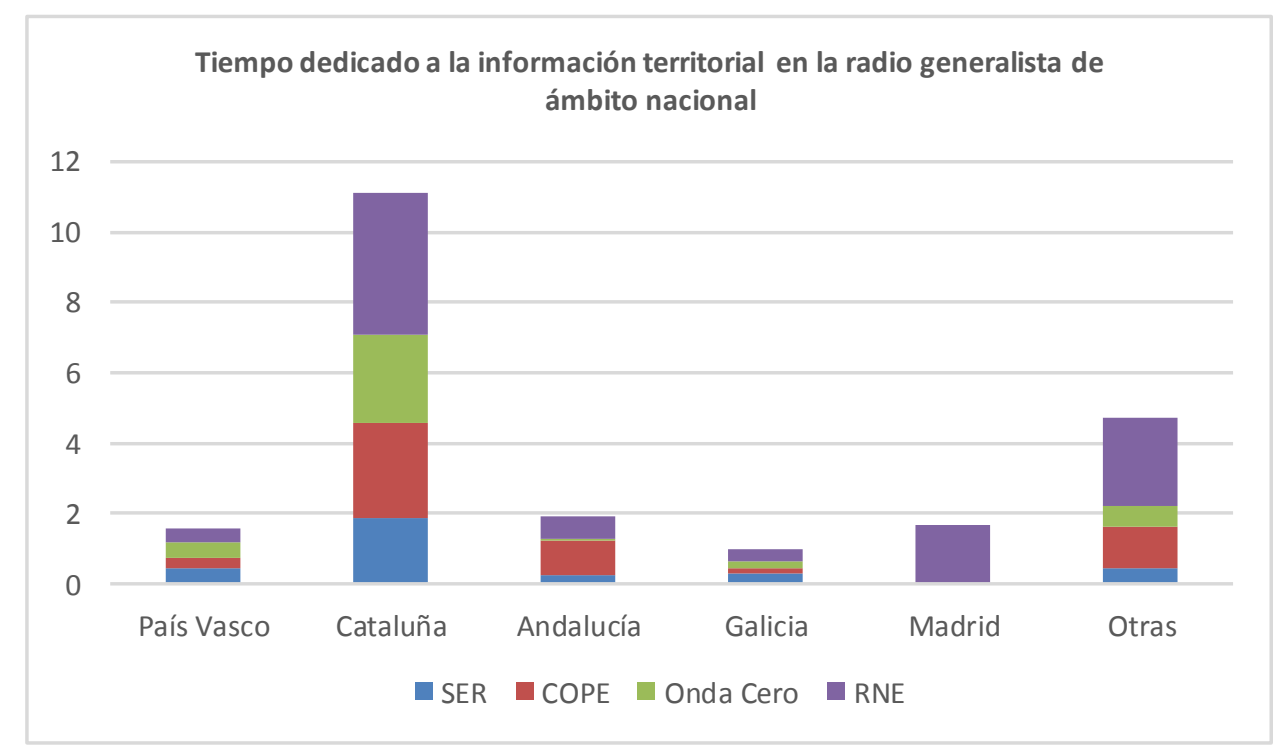

Fuente: elaboración propia.

\subsubsection{Hora 14, Cadena SER}

La Cadena SER, con 4.200.000 oyentes (31,1\% de share), es la emisora generalista española más escuchada en enero de 2017. El informativo Hora 14, presentado por José Antonio Marcos, tiene una audiencia superior a los 550.000 oyentes diarios (EGM, 2017). La estructura interna del espacio muestra una separación entre los tradicionales grandes bloques [7] informativos. Comienza con una batería de titulares de 6-7 minutos, donde se incluyen una media de 12 titulares, a la que sigue la desconexión territorial con la que concluye la primera media hora de programa; a continuación, se repite la batería de titulares, con leves variaciones, y se procede a desarrollar las principales noticias del día (unas 10) [8]. En los últimos 5 minutos de programa se repasan, en formato breve, los 3 acontecimientos más importantes del día y se despide el programa, generalmente, con piezas dedicadas al ocio y la cultura.

Por temática informativa, son las noticias de política e internacional las que ocupan la mayor parte del minutado (un 80\%), con lo que se trata de un espacio marcadamente político. El tratamiento de los hechos a los que se alude en el informativo es interpretativo, tanto por parte del conductor del 
programa como de los reporteros [9] a los que da paso, debido al uso de abundante adjetivación y al tono en que los presenta.

Respecto a la información perteneciente a las distintas comunidades autónomas, existe un relativo equilibrio entre todos los territorios. La comunidad más presente es Cataluña, que dobla en número de piezas a las siguientes, Madrid y Andalucía. Sin embargo, la cobertura de la reunión de Presidentes Autonómicos, así como distintos casos de corrupción menos mediáticos y los datos de accidentes de tráfico permiten que el resto de regiones tengan presencia, aunque sea más reducida. En el caso de Cataluña, su falta de entendimiento con el Gobierno central, los casos de corrupción y la inestabilidad del pacto formado por JxSí y la CUP [10] propician un tratamiento marcadamente negativo, con abundante adjetivación: "ausencia", "cicatería" (17/01/2017), "legalidad", "respeto", "radicalidad" (02/02/2017).

La información de tribunales en otras regiones, por el contrario, reciben un tratamiento discreto y sin valoraciones, como la causa abierta por el ático de Ignacio González (25/01/2017) o la imputación del presidente de Murcia (02/02/2017), que no pasan de titulares, o los casos de corrupción en el Ayuntamiento de Alcalá de Henares (09/01/2017) o en la delegación del sindicato UGT en Asturias (17/01/2017), relegados a los últimos puestos del informativo.

El resto de comunidades autónomas tienen una presencia testimonial: El País Vasco aparece relacionado con la dispersión de los presos de ETA (09/01/2017) y la ausencia de Iñigo Urkullu [11] en la Cumbre de Presidentes Autonómicos (17/01/2017); Galicia aparece vinculada al fuerte temporal que sufre en el mes de enero, y Castilla y León con dos informaciones referidas a los buenos resultados escolares obtenidos en el informe PISA $2016(09 / 01 / 2017)$ y al sobrecoste de las tasas universitarias en esta Comunidad Autónoma (25/01/2017). No obstante, en esta última noticia, Castilla y León comparte espacio con Cataluña, Madrid y Andalucía.

\subsubsection{Mediodía COPE, Cadena COPE}

Cadena COPE, con 2.711.000 oyentes (20,1\% de share) es la segunda emisora generalista española más escuchada en 2017. El informativo Mediodía COPE, conducido por José Luis Pérez y Pilar Cisneros, tiene una audiencia cercana a los 200.000 oyentes, una vez que aumentara en 2016 más de un $48 \%$ sus datos con respecto a 2015 (EGM, 2017). La estructura interna del espacio de noticias no se rige por la separación entre grandes bloques informativos, pero el formato es semejante al de las otras cadenas de radio. El informativo generalista de ámbito nacional tiene una duración de una hora que se divide en dos espacios de 30 minutos; en el primero, se incluyen un comentario del editor sobre la noticia más destacada del día, una batería de titulares que oscila entre los 10-11 diarios [12], el desarrollo de 3-5 noticias destacadas sin distinción explícita de su carácter nacional o internacional (aunque van consecutivas y en ese orden), las noticias más destacadas de la Comunidad de Madrid [13], los deportes y una conexión con la DGT para la última hora del tráfico. En el segundo bloque se amplían algunas de las noticias que han abierto el informativo a las 14 horas y se van introduciendo, a modo de sumario, otras informaciones de la actualidad, donde tienen presencia la economía, el Gobierno, noticias culturales y sucesos, además de publicidad y autopromociones de la cadena. Las noticias de sucesos y las de carácter internacional son las más abundantes en los informativos. El número de noticias por informativo no suele exceder de las 20.

En el tratamiento de la actualidad, destaca la importante dosis de interpretación de algunas noticias, con abundante adjetivación en muchos casos, y comentarios explícitos por parte del conductor del programa en las informaciones más destacadas. El enfoque prioritario que parece pretenderse es el de 
un punto de vista de "interés humano" en los acontecimientos y a partir de este se profundiza más en las noticias. Esto provoca que en el orden de exposición de las informaciones se muestren primero las que poseen víctimas de agresiones, accidentes o ataques, o aquellas personas que sufren las consecuencias de alguna negligencia administrativa o medida institucional, política, económica o natural. Los recursos más habituales son el paso a la crónica de calle y de redacción y la inclusión de declaraciones de los protagonistas de los hechos donde se acostumbra a encontrar tanto voces ciudadanas como fuentes oficiales.

En cuanto al protagonismo de las entidades territoriales autonómicas destaca, por encima de todas, Cataluña que dobla en presencia informativa a las dos siguientes, Madrid y Andalucía. También tiene una presencia, en cierto modo, significativa Galicia, aunque en este caso alude a referencias políticas ligadas al Gobierno del Partido Popular, a sucesos o a las inclemencias meteorológicas de la llegada del invierno. Por lo que respecta a Cataluña, y con un enfoque claramente negativo, las informaciones inciden en la brecha que separa al Gobierno catalán del español con motivo del proceso independentista y en señalar que las actuaciones del actual presidente catalán -Carles Puigdemont- y las instituciones autonómicas son interesadas y están ligadas al independentismo "pura ideología" (25/01/2017)- y en que son deficientes para con los ciudadanos de esta comunidad y del resto del Estado ("no tienen ni un grado de humanidad", "son presumidos", "despreocupados por los problemas ciudadanos"; 25 de enero de 2017).

El resto de las comunidades autónomas aparecen relacionadas con sucesos, principalmente (casos de Andalucía [14] y Galicia), el tiempo meteorológico o cuestiones anecdóticas. El País Vasco tiene presencia puntual en noticias de corte político como la Cumbre de Presidentes Autonómicos en el Senado y el nombramiento del nuevo Delegado del Gobierno, en las que se subrayan los "parecidos razonables" con el Gobierno independentista catalán, señalando que "tendrán que rendir cuentas a sus ciudadanos" por lo que no han hecho por ellos [15].

\subsubsection{Noticias Mediodía, Onda Cero Radio}

Onda Cero ocupa la tercera posición entre las emisoras generalistas españolas más escuchada en 2017 con un 14,9\% de share y 2.012.000 oyentes. El informativo Noticias mediodía, presentado por Elena Gijón y, en ocasiones, por Rafael Noblejas, tiene una audiencia de 204.000 oyentes (EGM, 2017). La estructura del informativo presenta dos bloques similares, uno de 20 minutos de duración y otro de 30, separados por una desconexión territorial. Ambos bloques comienzan con una batería de titulares, habitualmente 12, y profundizan en el relato diario con unas 14 piezas. No obstante, estos espacios no son idénticos, y se perciben cambios tanto en titulares como en noticias de una sección a otra. Incluye además dos menciones a la encuesta de la página web de la cadena y un espacio de opinión, ambas en clave de política nacional e internacional.

Las noticias de política predominan en el espacio informativo, llegando a ocupar más del doble que el resto de secciones juntas. El relato de actualidad se completa con internacional y economía, dejando muy poco espacio para noticias culturales, de sociedad y de sucesos. En cuanto al tratamiento de los hechos, este es muy interpretativo y utiliza una profusa cantidad de adjetivos en la mayoría de los casos. El enfoque utilizado es generalmente negativo cuando se refiere a informaciones de Tribunales, del Gobierno y de la llamada "cuestión catalana". Los recursos utilizados favorecen el posicionamiento ideológico del medio y, como en otros casos expuestos, hay una clara mayoría de declaraciones institucionales o de autoridades políticas frente a la escasa presencia de testimonios ciudadanos. 
Respecto a la presencia informativa de las distintas autonomías del Estado, Noticias mediodía focaliza la atención sobre Cataluña que, con 10 piezas en el periodo observado, suma el mismo número de noticias que el resto de comunidades juntas y ocupa siempre un lugar destacado en la escaleta. La imagen de Cataluña viene marcada por su enfrentamiento con el Gobierno central, los casos de corrupción y la inestabilidad del pacto formado por JxSí y la CUP, lo que acentúa un tratamiento muy negativo, con abundante adjetivación: "relevo obligado", "guerra interna", "fin de ciclo" (09/01/2017), "pérdida de tiempo y dinero", "ridículo" (25/01/2017), "manejos", "banda organizada", "ilícito" (02/02/2017), "desobediencia", "ruptura" (10/02/2017).

Con una menor incidencia aparece el País Vasco, protagonista negativo de la Cumbre de Presidentes Autonómicos por la ausencia de su representante. Además, el nombramiento del nuevo Delegado del Gobierno sirve para que la vicepresidenta del Gobierno, Soraya Sáenz de Santamaría, haga un llamamiento a la colaboración y el entendimiento, insinuando que las reticencias provienen de la Lehendakaritza.

El resto de regiones tienen una presencia testimonial, vinculada a la reunión de Presidentes Autonómicos, donde se da voz a Castilla y León, Murcia y Extremadura, y se menciona indirectamente a Canarias y Andalucía. En el espacio meteorológico se alude a zonas más que a regiones, o a localidades concretas; son habituales Galicia y Castilla y León.

\subsubsection{Diario de las 2, Radio Nacional de España}

RNE tiene 1.200.000 oyentes en 2017, lo que supone un 8,9\% de share. El espacio Diario de las 2, dirigido por Fernando Martín, contabiliza una audiencia en torno a los 300.000 oyentes, con una tendencia creciente durante el ejercicio anterior (EGM, 2017). La estructura que presenta el informativo es lineal, comenzando con una batería de titulares de 6-7 minutos, donde se incluyen una media de 10 titulares, y posteriormente se da paso al relato de la actualidad, con 36 piezas, sin espacio para la opinión y, de manera frecuente, con una breve entrevista a personalidades de la actualidad política. La duración del informativo es de 1 hora y no cuenta con desconexión territorial.

Las noticias de política y deportes son las informaciones con más tiempo en emisión, si bien el reparto de tiempos es mucho más equilibrado que en el resto de informativos radiofónicos analizados. Destaca la amplia cobertura de temas relacionados con el ocio y la cultura, fruto de la amplia red de centros territoriales y de la propia estructura del informativo que favorece su inclusión. El tratamiento de los hechos es fundamentalmente informativo y tanto el conductor como los corresponsales huyen del tono crítico e interpretativo que queda reservado para las declaraciones de los totales, aunque en menor proporción respecto a otras emisoras. El enfoque general más utilizado es el neutro, tanto por la baja adjetivación como por la variedad de versiones a las que se ofrece el micrófono.

En cuanto al reparto por autonomías, Cataluña, con 15 noticias, es la primera opción más representada [16], seguida de Madrid con 5 y Andalucía con 4. El tratamiento de la actualidad sobre Cataluña se centra en el apartado político, con el desafío independentista, las diferentes causas judiciales y, a diferencia del resto de emisoras, con piezas menores pertenecientes al ámbito de la cultura y sociedad. El enfoque que predomina es el neutro, gracias a la escasa adjetivación y a la contraposición de declaraciones, con un reparto de tiempos equilibrado.

El resto de las comunidades autónomas tiene una escasa presencia en el apartado político y siempre aparecen en bloque, como la Cumbre de Presidentes Autonómicos (17/01/2017) o la renovación del Tribunal Constitucional (25/01/2017). Cuando se muestran de forma individual, con cierto 
protagonismo, lo hacen en secciones como sociedad, cultura o sucesos. Así, en Andalucía destaca el juicio de Mercasevilla (17/01/2017); en Galicia los daños causados por un temporal (02/02/2017); en el País Vasco una exposición en el Guggenheim (02/02/2017) y en Castilla y León, los resultados del informe PISA (09/01/2017) y el precio de las tasas universitarias (25/01/2017)

\subsection{Análisis de los resultados obtenidos en los canales de televisión}

De los canales de televisión objeto de estudio, Telecinco es el que cuenta, dentro de sus informativos, con un mayor número de noticias referidas a las comunidades autónomas. En total, durante el periodo analizado, se contabilizan en esta cadena 65 piezas sobre noticias territoriales, frente a las 52 ofrecidas por TVE y las 39 de Antena 3, lo que supone una media de 13 informaciones de este tipo diarias. Este dato contrasta con el hecho que, de los tres, el espacio informativo de Telecinco es el que tiene una menor duración, que ronda los 35 minutos, mientras que el informativo de TVE se sitúa en torno a la hora de duración y el de Antena 3 alcanza los 40 minutos, con lo que la presencia de noticias regionales es aún más destacada.

\section{Gráfico 3. Peso informativo de las noticias territoriales en las cadenas de televisión}

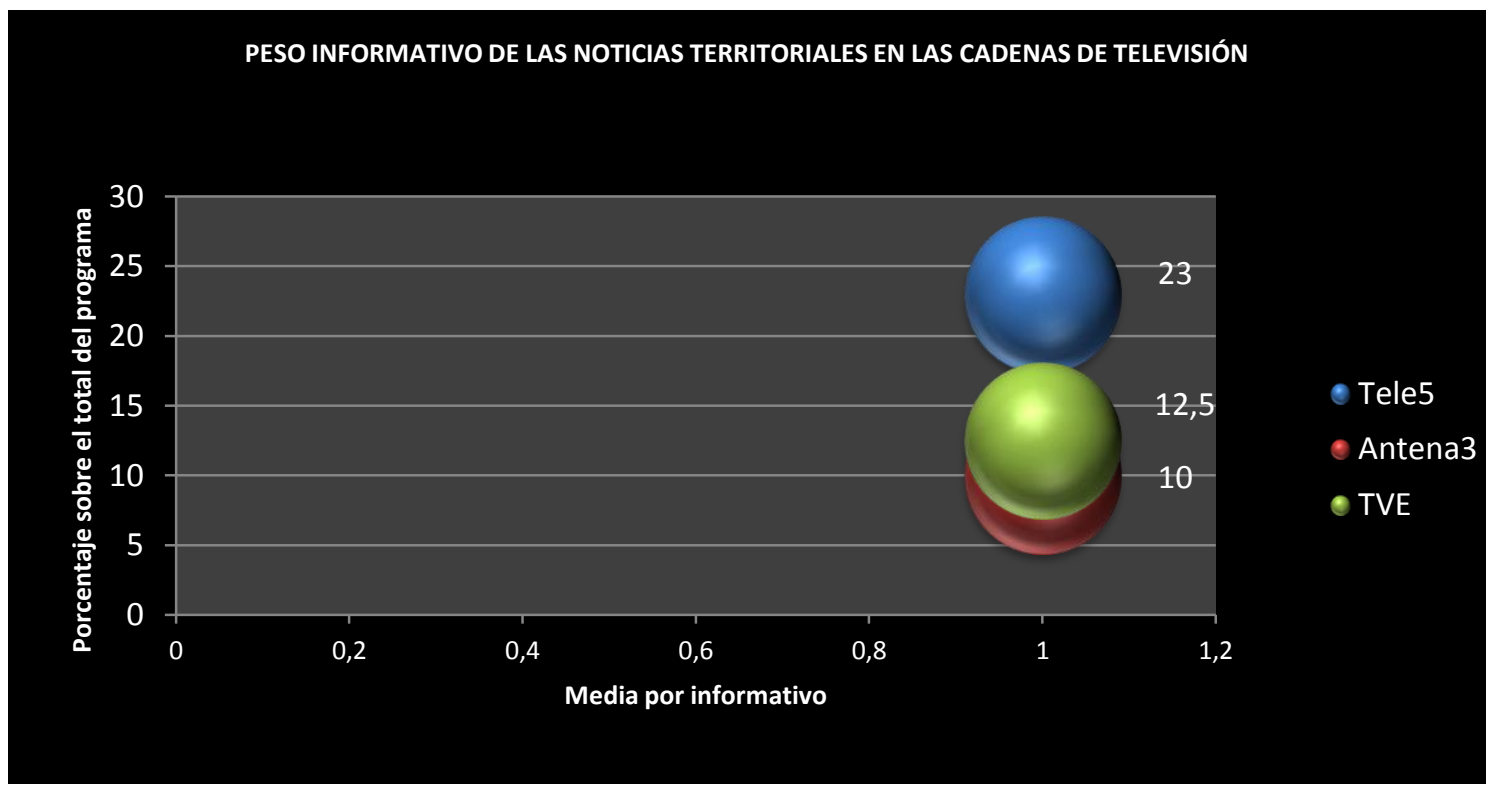

Fuente: elaboración propia. 


\section{Gráfico 4. Tiempo dedicado a la información territorial en la televisión generalista de ámbito nacional}



Fuente: elaboración propia.

Por comunidades autónomas, Andalucía es el territorio al que mayor espacio dedican, con un total de 17 piezas y alrededor de 20 minutos de emisión. Aunque todos los días de la muestra cuentan con alguna pieza, es el 17 de enero la jornada que cuenta con un mayor número de informaciones, un total de seis, seguida del día 10 de febrero, con cuatro piezas. La mayoría de las informaciones sobre Andalucía en Telecinco tienen relación con algún suceso, siendo minoritarias aquellas referidas a asuntos políticos o económicos autonómicos, algo que va en sintonía con el peso que los sucesos tienen en el reparto temático realizado en los informativos de este canal. De hecho, la sección sucesos es a la que más tiempo y noticias dedican, con una media de ocho minutos diarios y seis piezas.

\subsubsection{Informativos Telecinco 1}

Los informativos de Telecinco registran en el mes de febrero de 2017 una audiencia de 2.061 .000 espectadores (Barlovento Comunicación, 2017). El espacio de las 15.00 horas cuenta con una duración en torno a los 35 minutos y contiene alrededor de 27 piezas, todas ellas dedicadas a información general, puesto que la deportiva se ofrece a continuación en un espacio independiente. Se trata de un informativo que, a diferencia de otras ediciones -como la emitida a las 08.00 horas-, y de los informativos de otras cadenas- como los de Antena 3 y TVE-, no cuenta con una batería de titulares, sino que abre el espacio con una o dos informaciones destacadas. El espacio es conducido por David Cantero e Isabel Jiménez.

En Informativos Telecinco 1 destaca el volumen de información que dedican a sucesos de diferente índole, entre los que sobresalen los accidentes de tráficos y los casos de violencia de género y agresiones.

En lo que a la información territorial se refiere, Andalucía es la comunidad autónoma que acumula un mayor número de noticias. Durante la semana analizada, la región está presente con un total de 17 piezas. Aunque todos los días analizados nos encontramos con algún apunte informativo, el 17 de enero es la jornada con un mayor número de informaciones, 6, seguida del día 10 de febrero con 4. La mayoría de ellas son sobre sucesos, en especial, accidentes de tráfico y las actuaciones de la 
Guardia Civil contra los narcotraficantes en la costa gaditana. La segunda Comunidad Autónoma con mayor presencia es Cataluña, a la que se le dedican 9 informaciones. En todos los casos se trata de informaciones negativas, sobre todo en las de carácter político, donde se pone el foco en las informaciones que dejan en evidencia las malas actuaciones, o una praxis incorrecta, por parte de los partidos independentistas. Sobre Castilla y León se emiten seis informaciones, relacionadas con asuntos políticos, como el abandono entre gritos e insultos de la sede del Partido Socialista en Valladolid, Soraya Rodríguez, las bajas temperaturas debido a la ola de frío y viento, el cierre del pozo petrolífero más antiguo del Estado, dos accidentes de tráfico y la posible reapertura de la Central de Garoña.

Galicia cuenta con cinco informaciones en las que se da voz a la gente de la calle, centradas en asuntos sociales y sucesos. En esa misma línea, encontramos las informaciones procedentes de la Comunidad Autónoma Vasca, donde de las tres piezas incluidas, dos de ellas se centran en las consecuencias del temporal de viento y las fuertes lluvias registradas, y la tercera sobre la muerte de una mujer arrollada por una barredora municipal. Destaca la ausencia de informaciones sobre política autonómica, especialmente si se tiene en cuenta que durante esos días se celebra la Cumbre de Presidentes Autonómicos, evento al que Euskadi, al igual que Cataluña, no acude. Junto a estas comunidades autónomas también está presentes Murcia, Baleares, Canarias, Cantabria, Aragón, Extremadura, Castilla-La Mancha, Melilla y Navarra. Algunas de ellas figuran únicamente una vez, como es el caso del registro de la sede de UGT en Oviedo o un incidente en el aeropuerto de Melilla, mientras que otros territorios están presentes en el informativo de forma habitual, como es el caso de Madrid.

Las únicas regiones que aparecen destacadas en titulares son Cataluña, Cantabria, Madrid y Valencia, cada una de ellas con un titular.

\subsubsection{Antena 3 Noticias}

Los informativos de Antena 3 alcanzaron, durante febrero de 2017, una audiencia de 1.848.000 espectadores en las ediciones de la sobremesa y noche de lunes a domingo, convirtiéndose en el tercer informativo de televisión más visto, por detrás de TVE y Telecinco (Barlovento Comunicación, 2017). El espacio de las 15.00 horas cuenta con una duración en torno a los 40 minutos. Durante ese tiempo se emiten alrededor de 28-30 piezas de información general, puesto que el tiempo dedicado a los deportes se sitúa a continuación, como un espacio independiente, a pesar de que en titulares se incluya uno de deportes. La estructura del informativo consta de una batería de titulares -normalmente 9 de información general más uno deportivo, que ocupan tres minutos del informativo, aproximadamente- para después comenzar el relato de la información general del día. Sandra Golpe y María Rey son las encargadas de conducir todo el informativo, a las que se suma Rocío Martínez al inicio del mismo, para dar el último titular, el deportivo. Por secciones, política e internacional son las que más tiempo ocupan dentro del espacio. De hecho, ambas secciones ocupan la mitad del informativo.

Respecto a la información territorial, Andalucía vuelve a ser la comunidad autónoma con mayor presencia en el informativo de la sobremesa de Antena 3. Se le dedican un total de 8 informaciones. La mitad de ellas son de política, mientras que el resto se reparten entre sociedad y sucesos. Todos los días analizados contienen alguna información de Andalucía. El 10 de febrero es el único que no contiene ninguna pieza política. De estas informaciones, dos cuentan con titular. En segundo lugar, al igual que en el caso de Telecinco, se encuentra Cataluña, con 7 informaciones. De ellas, 6 noticias son sobre política autonómica. Además, se trata de informaciones que siempre tienen como 
protagonista a agentes que forman parte, o han formado parte, del Govern, como el primer aniversario de Carles Puigdemont en el Gobierno o el juicio por la celebración del 9N, y con las que generalmente se transmite una percepción negativa de la política autonómica. En este sentido, destaca la inclusión de una pieza en la que se incide en las faltas de ortografía de la carta que el presidente catalán le envía a Mariano Rajoy para explicarle su ausencia de la Cumbre de Presidentes Autonómicos. De estas informaciones, dos de ellas se destacan en titulares.

Galicia está presente con dos informaciones, ambas económicas, y el País Vasco cuenta con una información relacionada con el comercio. También en este caso, al igual que en Telecinco, llama la atención que este informativo no incluya ninguna información sobre la política autonómica, ya que ni siquiera dedican una pieza, tal y como hacen con Cataluña, para hablar de la ausencia de Euskadi en la Cumbre de Presidentes Autonómicos. Otras de las regiones presentes en Antena 3 Noticias son Madrid, con un total de cinco informaciones, así como Murcia, Valencia, Navarra, Extremadura, Asturias, Castilla y León, Canarias, Baleares y Aragón.

\subsubsection{Telediario 1}

La primera edición del Telediario de TVE cuenta con una audiencia de 2.029.000 espectadores que lo convierten en el informativo líder de audiencia en la franja horaria de la sobremesa (Barlovento Comunicación, 2017). El espacio comienza a las 15.00 horas y cuenta con una duración que oscila entre los 55-60 minutos, siendo el informativo más largo de los tres analizados en televisión. De ese tiempo, en torno a los 47-50 minutos, están dedicadas a la información general y el resto a la información deportiva. Es el único informativo de los tres que incluye los deportes en el informativo. Por piezas, la información general congrega en torno a 30-32, mientras que la información deportiva se compone de 6-8 noticias. Muchas de esas, además, son más largas que las emitidas, por ejemplo, por Informativos Telecinco, lo que le confiere un ritmo más pausado al informativo, en general. Es también el único informativo que comienza la emisión con una cabecera de entrada -los demás comienzan directamente con la que consideran la noticia o imagen del día- y cuenta con una única presentadora para información general, Pilar García Muñiz, a la que se suma un segundo presentador, Sergio Sauca, cuando llega el tiempo de los deportes. Al igual que en el caso de Antena 3 , se trata de un informativo que prima la información política y la internacional. Ambas secciones constituyen las temáticas a las que más tiempo dedican.

Si nos centramos en la información territorial, Cataluña es la comunidad autónoma con una mayor presencia en los informativos de TVE. Durante el periodo analizado cuenta con 14 piezas, a las que se suma una compartida con Euskadi: la referida a la ausencia de esta comunidad en la Cumbre de Presidentes Autonómicos. Todos los días cuenta con al menos una o dos informaciones. Aunque la sección de política es la que aglutina el mayor número de piezas, también aparecen varias sobre sucesos y sobre los efectos del temporal de viento, frío y lluvia. Cuatro de estas informaciones son destacadas en titulares. Al contrario de lo que ocurre en otros informativos, Andalucía cuenta con una presencia muy pequeña en los informativos de la televisión pública. Únicamente se contabilizan 3 noticias, 2 relacionadas con un mismo suceso, el caso de Marta del Castillo, y la otra de política. Ninguna de ellas figura en titulares. Galicia aparece en 3 informaciones - una de sucesos y otra de sociedad-, más otra compartida con Madrid sobre accidentes de tráfico. Ninguna de las 3 está en titulares. El País Vasco aparece dos veces con 2 noticias propias y una tercera en una noticia compartida con Cataluña. Dos de ellas están relacionadas con la política autonómica, mientras que la tercera pieza se incluye en sociedad. También con dos noticias aparece Castilla y León, una relacionada con las bajas temperaturas y otra con el cierre del pozo petrolífero más antiguo del Estado. El resto de comunidades autónomas son Madrid, Valencia, Navarra, Murcia, Aragón. De ellas, todas, a excepción de Murcia, aparecen en titulares. 


\section{Discusión de resultados y conclusiones}

Tras la exposición de los resultados obtenidos y su análisis, podemos resumir una serie de ideas fundamentales para la reflexión en función de los hallazgos descritos.

En primer lugar, nos encontramos con que las cadenas de radio generalistas ofrecen menos información territorial que las cadenas de televisión en sus informativos de mediodía y muestran importantes diferencias en el tratamiento de estas noticias. Asimismo, existen grandes desigualdades en el peso específico que se les otorga a las distintas comunidades autónomas, destacando por encima de todas ellas Cataluña, en el caso de la radio, seguida de Andalucía y Madrid; y Cataluña y Andalucía en el caso de la televisión, seguida por Madrid. En estas comunidades autónomas es donde tradicionalmente se concentran el poder político y económico del país, pero sobre todo destacan por generar el mayor número de noticias relacionadas con el ámbito parlamentario y los tribunales a raíz de los recientes acontecimientos ligados a la corrupción política, el proceso independentista catalán y los escándalos financieros. El resto de las comunidades aparecen, por lo general, sin protagonizar hechos noticiables de interés en los ámbitos de mayor nivel informativo: de la mano del contexto meteorológico, del estado de las carreteras, las pistas de esquí o el estado de las playas, el crecimiento del turismo o la celebración de algún evento cultural. Cuando estas comunidades son noticia la mayoría de las veces es debido a un suceso luctuoso, una agresión violenta, un accidente de tráfico o hechos similares. Por el contrario, Cantabria, La Rioja, Aragón o Castilla y León tienen una presencia testimonial en los informativos. El comportamiento de las cadenas de radio y de televisión en este punto es muy similar.

Otra de las diferencias encontradas entre la información territorial ofrecida en radio y televisión es que, mientras en el primer caso es la emisora pública la que cuenta con el mayor número de noticias territoriales, siendo, además, el informativo más largo de los cuatro analizados, en la televisión es el informativo de menor duración, y el de Telecinco, el que emite un mayor número de informaciones territoriales.

Por lo demás, el tratamiento en profundidad de las noticias territoriales, el enfoque, los recursos y fuentes utilizadas, la duración y el protagonismo que se le concede en la estructura de los programas informativos es semejante en los ámbitos radiofónico y televisivo, aunque son más abundantes las fuentes y recursos utilizados en las televisiones.

La imagen que difunden estos medios de comunicación nacionales de las comunidades autónomas es dispar y responde a sus respectivas líneas ideológicas, pero sigue un patrón común que se pone de manifiesto en la presentación de los contenidos particulares y el enfoque periodístico; así, Cataluña es tratada permanentemente con abundante adjetivación por parte de los conductores de los informativos y reporteros, empleando un tono crítico en el que la variable nacionalistaindependentista aparece siempre, aunque se hable de un accidente de tráfico, ofreciendo una imagen negativa del territorio y más en concreto del Gobierno de la comunidad. Lo mismo ocurre con Madrid cuando se abordan noticias relativas a su gobierno municipal. Ambas regiones, Cataluña y Madrid, son las que cuentan con presencia diaria en los informativos de radio y radio y televisión. En el resto de comunidades autónomas, dada que su aparición en los informativos se debe, fundamentalmente, a noticias relacionadas con sucesos - como es el caso de accidentes de tráfico y violencia de género-, sociedad -con cuestiones vinculadas a asuntos meteorológicos- y escándalos de corrupción política a nivel autonómico - como es el caso de la implicación del presidente de Murcia en el 'Caso Auditorio'-, su inclusión en los informativos, tanto de radio como de televisión, no es 
continua, sino esporádica, dado que dejan de aparecer en los servicios informativos tan pronto como la noticia "muere".

Nos encontramos, por lo tanto, que son las regiones con mayor peso económico y social -Cataluña y Madrid- las comunidades autónomas que aportan a los informativos mayor número de noticias políticas, seguidas de Andalucía, mientras que el resto sólo aparece en los informativos con noticias relacionadas, sobre todo, con sucesos y sociedad.

* Proyecto Programa de I+D+I Orientado a los Retos de la Sociedad, Ministerio de Economía y Competitividad, IP Ana Azurmendi, Transformación de la televisión autonómica: debilitamiento del servicio público y perspectivas de desarrollo tecnológico en un entorno de crisis, CSO2013-42270-R.

\section{Notas}

[1] Según los datos del último estudio del EGM publicado ( $3^{\text {a }}$ Ola de 2016) la televisión generalista en España, de lunes a domingo, muestra una audiencia de 38.627.000 individuos entre La 1 y Antena $3 \mathrm{TV}$, a los que habría que sumar los espectadores de Telecinco que no se contemplan en el EGM. El índice de penetración del medio es del 87,8\% en 2016. En radio, la audiencia de las cadenas generalistas objeto de este estudio, de lunes a viernes en el mismo periodo, es de 10.427.000 oyentes. El índice de penetración social de la radio en 2016 ha sido del $60 \%$.

[2] La presente aportación se inscribe en el marco del Proyecto de Investigación titulado "Transformación de la televisión autonómica: debilitamiento del servicio público y perspectivas de desarrollo tecnológico en un entorno de crisis. Impacto de Internet en la redefinición del servicio público de las televisiones autonómicas españolas" (MINECO, 2013-2017-CSO2013-42270-R), dirigido por Ana Azurmendi Adárraga, y está apoyado por el estudio "Nuevas formas de consumo, creación y producción informativa y otros contenidos audiovisuales", que lleva a cabo el Observatorio del Ocio y el Entretenimiento, (Cód.: OCENDI07/1618).

[3] Hay que tener en cuenta que estos horarios son abiertos ya que en el caso de los informativos principales de las 15 horas de las cadenas de televisión nacionales no todos duran una hora, sino que oscilan entre los 40 y los 60 minutos de lunes a viernes.

[4] No tendremos en cuenta las noticias presentes en las desconexiones territoriales por razones obvias.

[5] En ningún caso vamos a estudiar aquí los espacios o programas de opinión de cada cadena, al igual que tampoco las noticias deportivas que constituyan espacio aparte de los informativos, tanto en radio como en televisión, que son la mayoría de los casos.

[6] Salvo en el caso de COPE en la que es el 17 de enero el día que más tiempo dedica a noticias autonómicas, en concreto al caso de la muerte de una niña en Blanes (Cataluña) con una duración de más de 3 minutos, sin contar el comentario inicial del editor (y que distorsiona la media del cómputo total de información territorial para esta cadena)

[7] Según Soengas (2003), un informativo radiofónico principal respondería a la siguiente estructura: breve comentario del editor, batería de titulares a dos voces, información ordenada según los bloques política, sociedad, educación, sanidad, economía, cultura, deporte e información de servicio, con noticias meteorológicas y del tráfico, despedida del editor. 
[8] El promedio de noticias desarrolladas es de 10, y el de titulares es de 12, por lo que hay informaciones que no se corresponden con los titulares. Este hecho ocurre, en mayor o menor medida, con todos los informativos de todas las cadenas analizadas.

[9] En el caso de los reporteros, el tono interpretativo llama menos la atención puesto que forma parte de su labor en la exposición de una crónica.

[10] Junts Pel Si es la coalición política que gobierna en Cataluña con el apoyo de Convergencia Democrática de Cataluña y Esquerra Republicana (presidente Carles Puigdemont, 2015).

[11] Iñigo Urkullu Rentería es Lehendakari (presidente) del Gobierno Vasco desde 2012.

[12] El orden de los titulares no siempre responde al orden de la exposición de las noticias y, a menudo, se informa de hechos que no están presentes en esa batería inicial.

[13] Es lo habitual, aunque no siempre sigue esta estructura, por ejemplo, en el caso del informativo del 17 de enero de 2017.

[14] Andalucía también tiene presencia con relación a actos políticos relacionados con la guerra interna del PSOE por encontrar un líder de cara a su próximo congreso federal en 2017.

[15] Informativo 17 de enero de 2017, sección política (1, 5:11”).

[16] En la ficha de análisis la opción "Otras" engloba a las comunidades autónomas que no son Cataluña, País Vasco, Galicia, Andalucía y Galicia, y en RNE supone la opción más representada con 21 piezas informativas. Sin embargo, considerada en solitario, es Cataluña la comunidad autónoma que sobresale en presencia informativa en todos los espacios estudiados.

\section{Bibliografía}

Barlovento Comunicación (2017a): Audiencias TV febrero 2017. Madrid: Barlovento Comunicación: http://www.barloventocomunicacion.es/blog/15-audiencia-mensual/151-informe-audiencias-tvfebrero-2017.html

Barlovento Comunicación (2017b): Análisis televisivo 2016. Madrid: Barlovento Comunicación: http://www.barloventocomunicacion.es/blog/147-analisis-televisivo-2016.html

M C Cañamero (2014): "Extremadura representada en los medios de comunicación. Intertextualidad y estereotipos”. En Historia y Comunicación Social, número 19, esp. Marzo, pp. 351-362: http://dx.doi.org/10.5209/rev_HICS.2014.v19.45138

Consejo Audiovisual de Andalucía (2009): Andalucía en la radio. Su presencia en los informativos de las cadenas nacionales. Sevilla: Junta de Andalucía http://www.consejoaudiovisualdeandalucia.es/sites/default/files/publicaciones/andalucia_en_la_radio - 0. pdf

N Couldry y A Hepp (2012): Media Cultures in a Global Age. A Transcultural Approach to an Expanded Spectrum The Handbook of Global Media Research, First Edition. Edited by Ingrid Volkmer. Blackwell Publishing Ltd. Published 2012 by Blackwell Publishing Ltd.

I Crespo, C Moreno e I Delgado (2003): "El estudio de las campañas electorales. Partidos, medios de comunicación y electores. En Crespo, I. (Ed.) Partidos, medios de comunicación y electores. Los efectos de la campaña electoral de 2000 en España, pp. 13-24. Buenos Aires: Planeta. 
J M Chomón Serna (2011): "La radio pública no refleja la realidad territorial”. Actas del III Congreso Internacional Latina de Comunicación Social - III CILCS - Universidad de La Laguna: http://www.revistalatinacs.org/11SLCS/actas_2011_IIICILCS/018.pdf

L A Escudero (2005): "El valor de la imagen: un determinante para los destinos turísticos". En VV.AA. Galicia en cartel. A imaxe de Galicia na cartelaria turística. Santiago de Compostela: Universidad de Santiago de Compostela. Pp. 220-227.

Estudio General de Medios (2016): Informe General de Audiencias: http://www.aimc.es/-DatosEGM-Resumen-General-.html

J J Igartua y C Muñiz (2007): Medios de comunicación, inmigración y sociedad. Salamanca: Universidad de Salamanca

M L Humanes (2011): "El encuadre mediático de la realidad social. Un análisis de los informativos en televisión. En ZER, Revista de Estudios de Comunicación", (11), Pp. 119-141.

http://www.ehu.eus/ojs/index.php/Zer/article/view/6072

Instituto Nacional de Estadística (2017):

http://www.ine.es/FichasWeb/RegComunidades.do?fichas=49\&busc comu=\&botonFichas $=$ Ir $+\mathrm{a}+\mathrm{la}+$ $\underline{\text { tabla+de+resultados }}$

A López Téllez y F A Cuenca García (2005): “Televisión e información: análisis de los criterios de televisión de calidad en los informativos de las cadenas nacionales". En Comunicar, (25), 2. http://www.revistacomunicar.com/verpdf.php?numero=25\&articulo=25-2005-137

N López Vidales, A Tapia López y L Gómez Rubio (2016): “Grado de cumplimiento de la función de servicio público en los programas de éxito de las televisiones autonómicas en España. El caso de Canal Sur, Aragón Televisión y Televisión de Galicia”. Congreso CICOM de televisiones autonómicas. Publicado en actas. Facultad de Comunicación de la Universidad de Navarra: https://congresocicom2015tvautonomicas.files.wordpress.com/2016/06/lopez-tapia-gomez.pdf

C Lozano, J L Piñuel y J A Gaitán (2010): “Las verdades implantadas en los titulares de prensa sobre temas de comunicación. Análisis de la construcción de discursos hegemónicos a partir de las autoreferencias hacia la comunicación. En Razón y Palabra, número 74.

N Luhmann (1996): Die Realität der Massenmedien. Westdeutscher Verlag, Opladen.

M McCombs (2004): Estableciendo la agenda. Barcelona: Paidós

L Pitts (2011): “Objectivity might be impossible, so we strive for fairness". En Austin AmericanStatesman, pp. 219-229.

E Prado y M Delgado (2010): "La televisión generalista en la era digital", en Telos: https://telos.fundaciontelefonica.com/url-direct/pdfgenerator?tipoContenido $=$ articuloTelos\&idContenido $=2010083011290001 \&$ idioma $=e s$

F C Ruiz Morales (2007): La imagen de Andalucía en los informativos de televisión en España. Sevilla: Centro de Estudios Andaluces. Consejería de la Presidencia. Junta de Andalucía.

J Soengas Pérez (2003): Informativos radiofónicos. Madrid: Ediciones Cátedra, S.A.

W Wagner (1994): "Fields of research and socio-genesis of social representations: a discussion of criteria and diagnostics”. En Social Science Information, 33, pp. 199-228. 
M Wolf (1987): La investigación de la comunicación de masas. Crítica y perspectivas. Barcelona: Paidós.

\section{Cómo citar este artículo / Referencia normalizada}

N López Vidales, L Gómez Rubio, D Vicente Torrico (2017): "La información territorial en la radio y la televisión de ámbito nacional en España y su contribución a la imagen de las comunidades autónomas". Revista Latina de Comunicación Social, 72, pp. 649 a 666.

http://www.revistalatinacs.org/072paper/1184/34es.html

DOI: $10.4185 / R L C S-2017-1184$

- En el interior de un texto:

... N López Vidales, L Gómez Rubio, D Vicente Torrico (2017: 649 a 666)...

o

... N López Vidales et al, 2017 (649 a 666)...

Artículo recibido el 17 de marzo de 2017. Aceptado el 18 de junio.

Publicado el 20 de junio de 2017 